\title{
Clone breeding and vegetative reproduction of tea plant (Thea sinensis) in the conditions of Azerbaijan
}

\author{
F.A. Guliyev ${ }^{1}$ \\ ${ }^{1}$ Lankaran Regional Scientific Center of ANAS, Lankaran, Azerbaijan \\ Corresponding author email: prof.fquliyev@mail.ru
}

\section{ABSTRACT}

Tea growing in Azerbaijan is concentrated on an area of 5.33 thousand square kilometers, located within the Lankaran and Astara districts, including the districts of Astara, Lankaran, Masally, Lerik, Yardimly and Jalilabad. About $90 \%$ of Azerbaijani tea is produced in Lankaran, near the southern border with Iran. Tea was first grown in the Caspian region of Azerbaijan in the 1880s. Due to its favorable climatic conditions, the region currently produces $99 \%$ of the Azerbaijani tea harvest. The cultivation of tea in the country is limited by soil and climatic conditions and the biological characteristics of plants. The article gives a general characteristic of tea plantations growing in the conditions of the Lankaran-Astara zone (southern part) of the Republic of Azerbaijan, which is relatively unfavorable for tea plants. The results of research on clonal breeding of tea are presented, to select new forms to increase productivity and product quality. It is noted that during seed propagation, tea bushes have a significant difference in morphological and biological characteristics. Tea plants differ in yield, the strength of shoots, quality indicators of raw materials, length of the growing season, generative activity, and other economic characteristics. The variability inherent in the tea plant can be successfully used for the selection and isolation of the forms most valuable in terms of breeding characteristics, and their further vegetative reproduction. The transfer of the tea-growing industry of the republic to a modern scientific basis by breeding and planting plantations with new high-yielding and high-quality cultivars - clones will contribute to the intensification of production.

Keywords: shoot intensity, biochemical parameters, yield, flushes, vegetative propagation, morphological characteristics

\section{INTRODUCTION}

Tea is a widespread drink. Its value for the human body is estimated by many properties: the ability to quench thirst, maintain a high tone of the body, promote vigor and work capacity of a person. Thanks to these and other positive properties of tea, which in the initial period of its use was only a Chinese national drink, now it has become an international industrial commercial consumer product. The beneficial properties of the tea drink have been described by numerous researchers. Improving the varietal composition of tea plantations is one of the most difficult and complex problems of the tea industry.

The plantings available in the tea economy of Azerbaijan are a mixture of various morphological groups and transitional forms between them. It is known that the original tea in Azerbaijan was represented by seeds and seedlings obtained from various regions of 
Georgia, China, etc. During seed reproduction, it gives offspring that is heterogeneous in several morphological and biological characteristics.

Lankaran-Astara subtropical zone is the main base of tea growing in Azerbaijan (Figure 1 and 2). The following districts are engaged in tea cultivation: Lankaran, Astara, Masalli, partly Lerik, and slightly grow tea in the Zagatala-Balakan zone of Azerbaijan. LankaranAstara zone belongs to the zone of humid subtropical climate of the Mediterranean type. It is characterized by mild winters, short springs, hot summers, long and warm autumn. In general, the main distinguishing feature of the climate of the Lankaran-Astara zone, in comparison with the tea-producing regions of Western Georgia, is a lower moisture supply, with its uneven distribution throughout the year. In the spring-summer period, only $28.2 \%$ of the annual (1200-1300 mm) precipitation falls. In the autumn-winter period, $71 \%$ of precipitation falls, which causes temporary waterlogging of the soil of certain areas. In this zone, there are certain edaphic conditions - instead of the red soils of Georgia, yellowpodzolic, yellow-earth mountain-forest, gley, swampy soils, less favorable in physical and mechanical properties and fertility, are common here, which created special difficulties in their development for tea (Sanikidze and Sardzhevladze, 1973; Cheruiyot et. al., 2007).

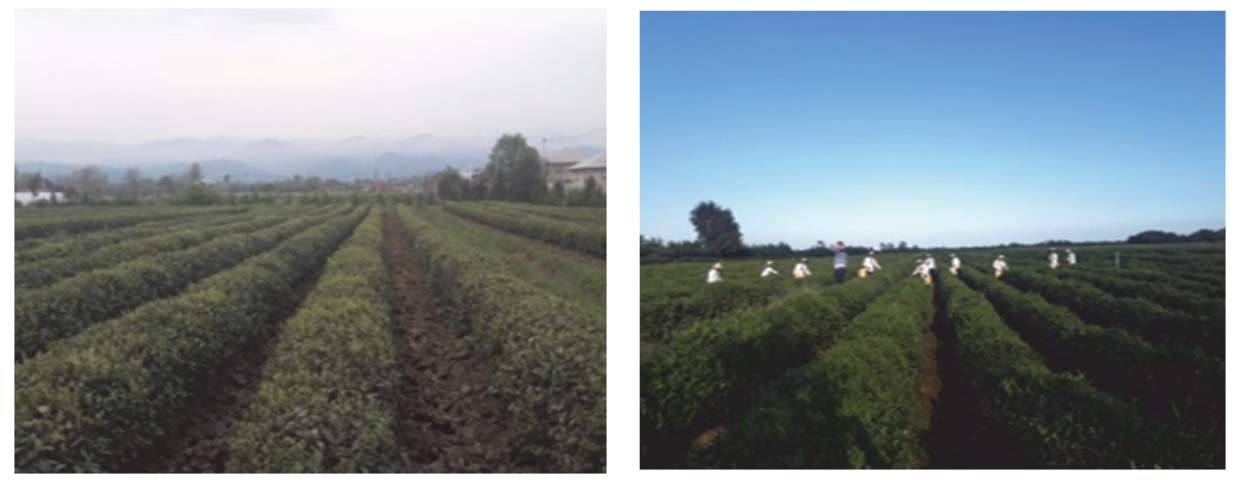

Figure 1-2. Tea plantations of the Lankaran-Astara zone of Azerbaijan (southern part of the country)

The mutual combination of meteorological indicators and soil conditions creates a kind of ecological environment that differs from other zones of the world tea growing. In this regard, the question arises of searching for other, more reliable methods of soil development and establishment of tea plantations concerning the climate characteristics of the humid and semi-humid subtropics of Azerbaijan. As a result of the unfolding research work in the Lankaran branch of the Azerbaijan Scientific and Production Association of Horticulture and Subtropical Crops (AzSPAHSC), this method was found.

As you know, one of the powerful factors for increasing the yield of tea plantations and improving the quality of products is the breeding of selected cultivars of tea, adapted to the local climatic conditions of cultivation (Bakhtadze, 1971; Guliev and Babaev, 1982; Gvasalia, 2015). The technology of tea cultivation in the subtropics of Azerbaijan should ensure longterm productivity, high yield, and high quality products, taking into account the main limiting factor - regulation of the water regime of the soil and plants, in combination with other links of plantation care.

Large studies in this direction were carried out by the employees of the Lankaran branch of AzSPAHSC and the Lankaran Regional Scientific Center of the National Academy of Sciences of Azerbaijan on such important issues of tea growing as biology, breeding, and seed production of tea. The main method in breeding work was the classical selection method selection of initial pairs according to a complex of traits, hybridization by additional pollination with a mixture of pollen, selection of hybrid seedlings according to the sum of 
positive traits, their improvement by selection and additional pollination, consistent in three generations.

In Azerbaijan, tea plantations are a mixture of various morphological groups of the Chinese and transitional forms between them. It is known that the original seed material of tea was brought to Azerbaijan from Georgia, which in turn was obtained from various regions of China, Japan, India, and Ceylon. Dilution of this material in several generations gave the tea, which is known in our country under the name of local-Azerbaijani. During seed reproduction, it splits, as a rule, it gives offspring heterogeneous in several morphological and biological characteristics (Makarova, 1936; Daraselia et al.,1989).

Thus, established tea plantations differ in the yield of tea leaves, the strength, and intensity of shoot formation, the energy of shoot growth, the length of the growing season, the nature of branching, the degree of generative activity, winter hardiness, drought resistance, and other economic characteristics. At the same time, tea bushes differ among themselves and in biological characteristics that determine the quality of the finished tea product (Tuov, 2010; Tuov and Ryndin, 2011). The variability of the tea genus creates the possibility of a wide selection of selectively valuable forms. Along with economically productive plants, lowyielding bushes grow on tea plantations, the presence of which is undesirable, since they create variegated plantings and make it difficult to properly care for the tea culture and reduce the yield and quality of the product (Bakhtadze, 1971); Gabrichidze, 1974; Kutubudze and Mutovkina, 2008; Gvasalia, 2015). The heterogeneity like the development and vegetation of tea bushes makes it difficult to use agrotechnical methods of care, especially with mechanized care and collection of leaves, in which the uniformity of the trellis in height and the simultaneous appearance of flushes are of great importance. The diversity of plantings in terms of quality also complicates technological processing and obtaining high quality products (Kolemishvili et al., 1941; Tuov et al., 2012).

The value of this study lies in the use of the variability that is inherent in the tea plant and the breeding, on its basis, of forms valuable in terms of economic characteristics and their further vegetative reproduction. Breeding new high-yielding, high-quality clones of tea will make it possible to transfer the tea-growing industry in the republic to varietal production and increase its efficiency. This complies with the requirements approved on February 18, 2018, by the State Program for the restoration and development of tea growing in Azerbaijan. The implementation of this program will significantly meet the needs of the country's population with high-quality domestically produced tea. In the Lankaran-Astara humid-subtropical zone of Azerbaijan, research on the propagation of tea by the vegetative method was carried out in the Lankaran branch of AzSPAHSC in the 70s of the last century.

Studied vegetative propagation of tea by cuttings. Rooting of tea cuttings was carried out, as was customary for tea-producing regions of the world, and in particular in Western Georgia, in the open field. These studies in Lankaran did not give positive results, due to the unfavorable conditions of the zone - arid climate, high air temperature, and low relative humidity. Under such conditions, tea cuttings could not form a root system, and attempts to regulate soil moisture by artificial irrigation and shading the cuttings did not give the desired result.

Thus, due to the unfavorable conditions in the Lankaran-Astara subtropical zone in the open field, it is almost impossible to obtain root formation during the vegetative propagation of tea by cuttings. In the years 1982, (Guliev and Babaev, 1982), and 1986, respectively (Babaev, 1986), the researchers in the Lankaran branch of AzSPAHSC tea had concerns related to the vegetative multiplication of tea. They developed and recommended a vegetative propagation through green cuttings in a soil substrate in polyethylene bags in rooms under the frame, in the presence of a steaming installation.

Currently, in modern literature, the issue of vegetative reproduction is well developed and this method is widely used on several crops, being most widely used in fruit growing. 
The first works on the vegetative propagation of a tea plant in Georgia were presented by Kvaratskhelia, (1930) at the Tea Institute, in the village of Anaseuli, Maharadze region of Georgia. He also started it back in 1928 at the Sukhum experimental station.

Vegetative propagation of tea in Georgia was carried out by Acad. Bakhtadze (1974) but also by authors (Mutovkina, 1974; Makarova, 1936; Kolemishvili, 1941; Gabricidze,1974; Bakhtadze, 1971; Kutubudze and Mutovkina, 2008; Gvasalia, 2015; Gvasaliya et. al., 2019). In the 1980s, the Scientific Research Institute of Tea and Subtropical Crops (Georgia) developed and recommended a method of vegetative propagation of tea by cuttings.

In some provinces of Japan (Shizuko, Kanaya), tea plantations with cuttings have long been used.

According to Gabricidze (1974) earlier in Ceylon, the method of vegetative propagation of tea was used for breeding individual bushes. A little later, in Ceylon, vegetative propagation by cuttings in plastic bags was also of industrial importance. New tea plantations or replanting of old ones was carried out by planting cuttings of their best zoned clones.

\section{MATERIALS AND METHODS}

Research on clone selection was started. as it was indicated in the 80s of the last century in the Lankaran branch of AzSPAHSC. The selection of plants on tea plantations, planted in the Lankaran-Astara zone by seedlings grown in the nursery (Figure 3 and 4).
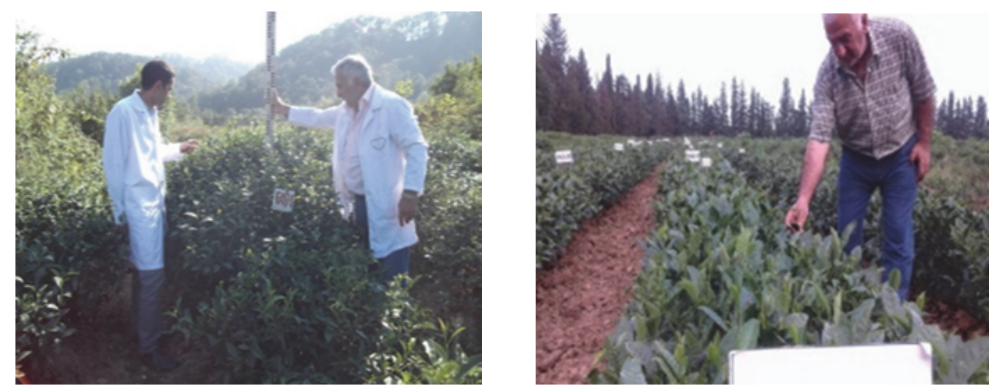

Figure 3 and 4 . Tea seedlings in the nursery

On the established tea plantations, the approbation of bushes and the selection of plants were carried out, differing in the yield of green tea leaves, the nature of the development of the habit of the bush, the intensity of shoot formation, the simultaneous approach of shoots to the collection, growth energy, the activity of shoot formation, the ability of the plant to give at least five ordinal shoots in the season of leaf collection. The assessment of the plant by yield was carried out by the weight of the harvested leaf during the entire growing season (Mutovkina, 1974; Ryndin and Tuov, 2006). The selection of bushes for quality was carried out by determining the chemical components in the tea leaf - tannin and extractives. A technological assessment of the finished product was given by the processing of raw materials. Research on the isolation of new clones of tea was carried out according to the following scheme: selection of initial plants, a study of their morphological characteristics, biology, yield, and quality indicators of raw materials and finished products. Analyzing, selecting, and studying the best bushes, propagating them by cuttings, checking clones at the experimental clone testing site, propagating the best clones to obtain a cultivar. State cultivar testing and introducing the best cultivars into production. The selection of initial plants was carried out on full-age and young tea plantations, established in the LankaranAstara zone of Azerbaijan, as well as on the territory of the breeding nursery of the Auxiliary Experimental Economy (PEC) of the Lankaran branch of AzSPAHSC. On the selected bushes, a morphological description was carried out according to all the main characters that 
determine the breeding value of plants. Biochemical studies were carried out at the end of the growing season. The size of the bush was determined by measuring the height and width of the crown in two diameters. The crown area was studied from the average crown diameter according to the formula $\mathrm{D}=3.14 \times \mathrm{d} 2 \times \mathrm{H} / \mathrm{H}$. The size of the leaf surface was determined by measuring the length and width, multiplied by a factor of 0.7 . For the description, mature leaves typical for a given bush were taken in an amount of at least 20-30 pieces per plant. The biology of the bush was studied concerning vegetation, budding, flowering, fruiting, the degree of drought resistance. The yield of the bush was established by systematic collection and weighing of the leaf during the entire growing season. In the collected leaf, the composition of two-three-leafed normal and deaf flushes was taken into account (Figure 5). To determine the quality of the product, harvests were taken once in the days of mass collection from each plant. Samples for analyzes were taken in $100 \mathrm{~g}$ fixation of the sample and biochemical studies were carried out in the laboratory of the Lankaran branch of AzSPAHSC. In the leaves, the content of total tannin and the number of extractives was determined as the main components that determine the quality indicators of tea (Dzhemukhadze et al., 1958). At the end of the study, the mother plants were singled out according to the main indicators of yield and quality. Selected bushes were propagated by cuttings (Kvaratskhelia and Erstavi, 1934; Kvaratskhelia,1930; Guliev and Babaev, 1982). Experimental plots of clone testing were laid with planting seedlings. In the clone test, clones were tested in homogeneous growing conditions for all indicators of economic value. The best clones, which were highly appreciated in terms of yield and quality, were subsequently propagated vegetatively by cuttings to obtain clone cultivars. The data of promising clones were compared with the control zone for the LenkoranAstara zone, the tea cultivar 'Azerbaijan 2'. The analyzed data represent averages of three replication. Data obtained for all analyzed indicators of tea were ensured statistically by calculating standard deviations.

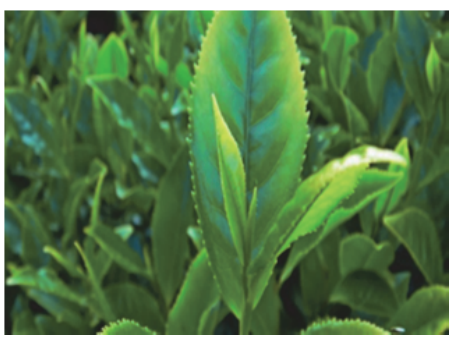

Figure 5. Normal flush

\section{RESULTS AND DISCUSSIONS}

The selection of the original mother plants, as mentioned above, was carried out on tea plantations, planted by seedlings grown in the nursery. The selected tea bushes were cloned within the breeding population for three years. They were propagated by vegetative cuttings and experimental plots for clone testing were laid in the Lankaran branch of AzSPAHSC.

Among economically valuable traits, particular importance was attached to the general development of the bush, a high capacity for shoots and large leaves. The drought resistance of plants and the general state of life were taken into account. Data on the study of the morphological characteristics of the mother bushes identified on the tea plantations of the zone are shown in Table 1.

As can be seen from Table 1, the selected plants by morphological characteristics, respectively, differed in positive characteristics from the rest of the bushes existing on the plantations. Table 2 shows the individual characteristics of the selected mother bushes.

As can be seen from table 2, the intensity of shoot formation, estimated in points in the selected mother bushes, is quite high, and ranges between $4.5-4.7$ points.

The weight of 3-leaf flushes is 1.2-1.3 g and the yield of green tea leaves from one bush is on average $372-410 \mathrm{~g}$ for the harvesting season, which corresponds to recalculation per hectare - 8134-9020 kg. The study of biochemical parameters (tannin and extractives) 
showed the content of tannin in 2-leaf flashes of 24.2-26.1\%, and of extractives 45.3 $48.2 \%$.

Table 1. Morphological characteristics of mother tea bushes, allocated on tea plantations in the Lankaran-Astara zone of Azerbaijan

\begin{tabular}{|c|c|c|c|c|c|c|c|}
\hline Tea plantation areas & 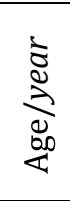 & 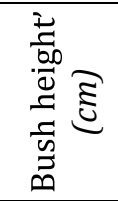 & 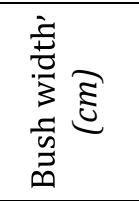 & 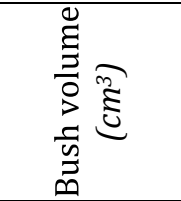 & 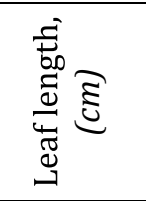 & 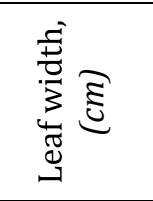 & 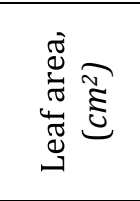 \\
\hline $\begin{array}{ll}\text { Lankaran, } & \text { Haftoni } \\
\text { village } & \end{array}$ & 21 & $80 \pm 2.1$ & $120 \pm 3.1$ & $90432 \pm 2.3$ & $10.0 \pm 0.7$ & $4.5 \pm 0.01$ & $31.5 \pm 2.8$ \\
\hline $\begin{array}{l}\text { Lankaran, Haftoni } \\
\text { village }\end{array}$ & 20 & $70 \pm 1.9$ & $120 \pm 2.9$ & $79128 \pm 3.4$ & $10.2 \pm 0.1$ & $4.5 \pm 0.01$ & $32.1 \pm 2.2$ \\
\hline $\begin{array}{ll}\text { Lankaran, } & \text { Hirkan } \\
\text { village } & \\
\end{array}$ & 19 & $70 \pm 1.4$ & $110 \pm 1.7$ & $66489 \pm 2.1$ & $10.1 \pm 0.3$ & $4.6 \pm 0.03$ & $32.5 \pm 1.4$ \\
\hline $\begin{array}{ll}\text { Lankaran,Khanbulan } \\
\text { settlement. } \\
\text { AzSPAHSC }\end{array}$ & 27 & $70 \pm 1.3$ & $110 \pm 1.2$ & $66489 \pm 1.6$ & $10.1 \pm 0.2$ & $4.4 \pm 0.07$ & $31.1 \pm 1.1$ \\
\hline
\end{tabular}

Table 2. The Intensity of shoot formation, the weight of three-leaf flushes, yield and quality of isolated mother tea plants

\begin{tabular}{|c|c|c|c|c|c|c|c|}
\hline Tea plantation areas & 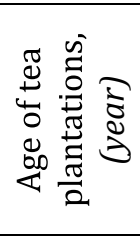 & 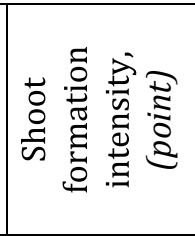 & 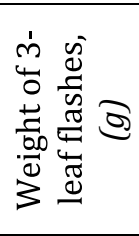 & 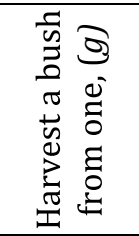 & 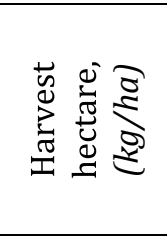 & 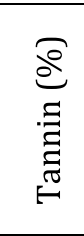 & 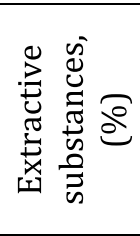 \\
\hline Lankaran, Haftoni village & 21 & $4.5 \pm 0.05$ & $1.3 \pm 0.3$ & $400 \pm 3.5$ & $8800 \pm 3.3$ & 26.1 & 48.2 \\
\hline Lenkoran, Havzava village & 20 & $4.5 \pm 0.04$ & $1.2 \pm 0.6$ & $410 \pm 3.7$ & $9020 \pm 3.9$ & 24.2 & 45.3 \\
\hline Lankaran, Hirkan village & 19 & $4.5 \pm 0.02$ & $1.3 \pm 0.1$ & $372 \pm 2.5$ & $8184 \pm 3.1$ & 25.2 & 47.1 \\
\hline $\begin{array}{lr}\text { Lankaran, } & \text { Khanbulan } \\
\text { settlement. } & \text { Experimental } \\
\text { AzSPAHSC } & \end{array}$ & 27 & $4.7 \pm 0.07$ & $1.2 \pm 0.4$ & $388 \pm 2.9$ & $8536 \pm 2.7$ & 26.1 & 47.4 \\
\hline
\end{tabular}

Table 3 shows the morphological characteristics of the isolated promising tea clones. The data given in the table confirm the high potential biological productivity of the studied clones and their superiority over the control plants (Table 3).

Table 3. Morphological characteristics of promising clones

\begin{tabular}{|c|c|c|c|c|c|c|}
\hline Promising clones & 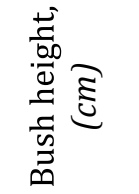 & 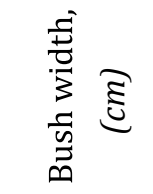 & 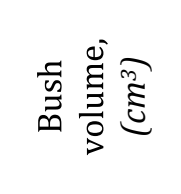 & 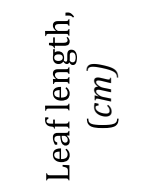 & 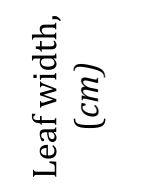 & 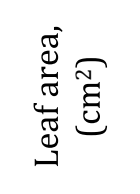 \\
\hline '1/73' & $90 \pm 2.8$ & $130 \pm 2.7$ & $11.939 \pm 1.1$ & $11.0 \pm 0.09$ & $4.5 \pm 0.03$ & $34.5 \pm 0.9$ \\
\hline '2/73' & $70 \pm 1.2$ & $130 \pm 2.04$ & $9.986 \pm 1.4$ & $11.1 \pm 0.04$ & $5.0 \pm 0.07$ & $38.5 \pm 0.1$ \\
\hline '3/34' & $70 \pm 1.6$ & $120 \pm 1.09$ & $7.928 \pm 1.8$ & $12.2 \pm 0.01$ & $5.1 \pm 0.01$ & $42.8 \pm 0.1$ \\
\hline '4/44' & $80 \pm 1.03$ & $120 \pm 1.03$ & $9.043 \pm 1.9$ & $11.0 \pm 0.03$ & $5.0 \pm 0.05$ & $38.5 \pm 0.2$ \\
\hline Control 'Azerbaijan-2' & $60 \pm 1.01$ & $90 \pm 1.05$ & $3.815 \pm 2.9$ & $8.4 \pm 0.01$ & $3.8 \pm 0.01$ & $22.8 \pm 0.3$ \\
\hline
\end{tabular}


In terms of the volume of the bush, there was a clear advantage of promising tea clones (7.928-11.939 $\left.\mathrm{cm}^{3}\right)$ in comparison with the control variant - the 'Azerbaijan 2' in which this figure is $3.815 \mathrm{~cm}^{3}$. The same applies to the leaf area: in the zoned control tea - 'Azerbaijan 2' $-22.8 \mathrm{~cm}^{2}$, in the isolated clones $-34.5-42.0 \mathrm{~cm}^{2}$.

Quite interesting data were obtained when studying the volume of the crown of bushes and plants of internodes. The studies have shown that in terms of height, diameter and overall volume of the bush, the selected clone are 2-3 times higher than the control variant 'Azerbaijan 2' (Table 4).

Table 4. Study of the volume of the crown of bushes and the distance between internodes

\begin{tabular}{|c|c|c|c|c|c|c|}
\hline \multirow[b]{2}{*}{$\begin{array}{l}\text { Promising } \\
\text { clones }\end{array}$} & \multirow{2}{*}{ 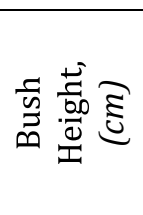 } & \multirow{2}{*}{ 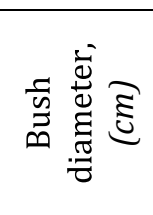 } & \multirow{2}{*}{ 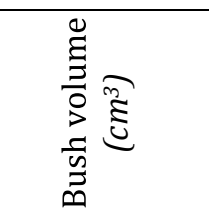 } & \multirow{2}{*}{ 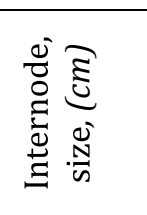 } & \multicolumn{2}{|c|}{$\begin{array}{l}\text { Comparison with } \\
\text { control }\end{array}$} \\
\hline & & & & & $\begin{array}{c}\text { Bush } \\
\text { volume, } \\
\text { (\%) }\end{array}$ & $\begin{array}{l}\text { Internode } \\
\text { size, } \\
(\%)\end{array}$ \\
\hline '1/73' & $90 \pm 0.01$ & $130 \pm 0.9$ & $1193.98 \pm 0.1$ & $6.0 \pm 0.8$ & 313.0 & 300.0 \\
\hline '7/18' & $90 \pm 0.01$ & $140 \pm 0.9$ & $1384.74 \pm 0.1$ & $5.0 \pm 0.1$ & 363.0 & 250.0 \\
\hline '6/44' & $80 \pm 0.04$ & $120 \pm 0.01$ & $904.32 \pm 0.4$ & $4.0 \pm 0.02$ & 237.0 & 200.0 \\
\hline '3/341' & $70 \pm 0.05$ & $120 \pm 0.01$ & $791.28 \pm 0.7$ & $5.0 \pm 0.05$ & 207.4 & 250.0 \\
\hline $\begin{array}{c}\text { Control } \\
\text { 'Azerbaijan-2' }\end{array}$ & $60 \pm 0.03$ & $90 \pm 0.04$ & $381.51 \pm 0.2$ & $2.0 \pm 0.05$ & 100.0 & 100.0 \\
\hline
\end{tabular}

Table 4 shows the results of a study to study the composition, number and mass of flushes of promising tea clones per unit area $\left(625 \mathrm{~cm}^{2}\right)$.

\section{CONCLUSION}

Long-term research work on clone breeding of tea began under the scientific guidance of prof. F. Guliyev since 1982 in the Lankaran branch of the Azerbaijan Scientific Research Institute of Fruit and Tea Growing. Methods for breeding clones have been developed. The main principle was: selection of valuable forms, the study of their yield, quality, vegetative propagation by green cuttings, clone testing, the establishment of plantations of mother plants of clones.

During long-term studies on vegetative propagation of tea and clonal selection, it is shown that in the specific conditions of the Lankaran-Astara zone of Azerbaijan - dry springsummer periods, high temperature, low relative humidity, based on the derived clone, vegetative propagation of the tea culture can be used.

Techniques for rooting tea with green cuttings in yellow-earth-podzolic soil in specific conditions of the Lankaran zone of Azerbaijan and growing seedlings in fog-forming chambers, planting on plantations at the age of 1-2 years in polyethylene bags have been developed.

Research in the Lankaran-Astara zone of Azerbaijan on clonal breeding of tea gave positive results. For the first time, new clone was bred: '1/73' - 'Farmanchay', '2/17' - 'Lankaran, '3/341' - Khazar, '6/44' - 'Farmanchaychakhrai'. During 2015-2019, they successfully passed the State Test and were zoned into production in the Lankaran-Astara zone of Azerbaijan.

It has been established that the clone cultivars 'Farmanchay', 'Lankaran', 'Khazar' and 'Farmanchaychakhrai' are characteristic for their high productivity in the specific conditions of the Lenkoran-Astara zone (12-15 tons/ha), high tannin content (28-30\%) and extractives (44-46\%) in raw materials and finished products, respectively 12.5-16.0; 12.5-13.8; 38.8 42.9 specific aroma and taste. 


\section{REFERENCE}

1. Babaev M.M., and Guliev F.A. (1986). Results of studying vegetative propagation of tea in the Lankaran zone. Journal "Subtropical cultures", №3, pp:48-51.

2. Bakhtadze K.E. (1971). Biological basis of tea culture, Tbilisi: Metzniereba, pp. 359.

3. Cheruiyot Ek, Lm Mumera, Wk Ngetich, A Hassanali and F Wachira (2007). Polyphenols as potential indicators for drought tolerance in tea (Camellia sinensis L), Bioscience, Biotechnology and Biochemistry, 71:21902197.

4. Daraselia M.K., Vorontsov V.V., Gvasalia V.P. and Tsanava V.P. (1989). Tea culture in the USSR. Tbilisi: Metsniereba., pp.533. ISSN 5-520-00355-6.

5. Dzhemukhadze M.K., (1958). Fundamentals of biochemical control of tea production. - M: Publishing house of the Academy of Sciences of the USSR. pp-166.

6. Gabrichidze Z.Sh. (1974). Progressive technology of vegetative propagation of breeding and clones of tea. Abstracts of the seminar on vegetative propagation of tea, Moscow;

7. Guliev F.A., and Babaev M.M., (1982). Results of studying vegetative propagation of tea in the conditions of the Lankaran zone of Azerbaijan. Journal "Subtropical cultures", Makharadze-Anaseuli. Collection Materials "All-Union coordination meeting on productivity.

8. Gvasalia M.V. (2015.). Spontaneous and induced cultivars and forms of tea (Camellia sinensis (L.) Kuntze) in the humid subtropics of Russia and Abkhazia, the prospects for their reproduction and in vitro preservation: author's abstract, Dis. Cand. Biol. Sci., Krasnodar, pp 24.

9. Gvasaliya M.V., Samarina LS, Choudhary R., Ahlawat J. and Yadav S.K. (2019). Genetic variability of tea plant on the Black Sea Coast of Caucasus. Seed Research 47(1): 72-76

10. Kerkadze I.G. (1980). Some questions of genetics of a tea plant, Communication VIII, Somatic mutation and clonal selection of tea, Subtropicheskie Kultury, 1:42-45.

11. Kerkadze I.G. (1987). Theory and practice of spontaneous and induced mutagenesis of subtropical crops: author's abstract. Disease Document of Biological Sciences, Moscow, pp- 51.

12. Kolemishvili M.V. (1941). Vegetative propagation of tea. VNIICHiSK Bulletin, №2, 1941, pp: 34-44.

13. Kutubudze B.B and Mutovkina T.D., 2008. Clonal breeding of tea. Monograph. Batumi-Chakva, (2008), pp.99

14. Kvaratskhelia T.K. (1930). A few words about the propagation of a tea bush by cuttings. Journal "Subtropical cultures", №2, pp: 16-23.

15. Kvaratskhelia T.K. and Erstavi D.Sh.(1934). Vegetative propagation of the tea bush. Journal "Proceeding VNIICHKh", №3, pp: 11-18.

16. Makarova K.N., (1936). Vegetative propagation of tea. Journal "Soviet subtropics", №4, pp: 28-36.

17. Mutovkina T.D. (1984). Clonal breeding of tea. Abstracts of the seminar on vegetative propagation of tea. Moscow, p. 30-36.

18. Ryndin A.V. and Tuov M.T. (2006). Cultivation of tea in the subtropics of Russia//Science of the Kuban.-- №4 pp:28-32. ISSN 1562-9856

19. Ryndin A.V. (2007). Breeding new cultivars of tea - an innovative direction of the breeding process // Gardening and viticulture. №3 pp:23-24, ISSN 0235-2591.

20. Tuov M.T. (2010). Breeding, introduction and study of tea in the subtropics of Russia // Subtropical cultures №1., pp: 38-42. ISSN 02079224.

21. Tuov M.T.; and Ryndin A.V. (2011). Results of the study of promising tea hybrids in the subtropics of the Russian Federation // Subtropical and decorative gardening, pp:101-109. ISSN 2225-3068.

22. Tuov M.T, (2012). Biology, breeding and modern assortment of tea in Russia//Subtropical and decorative gardening. T. 46- №1, pp:114-122. ISSN 2225-3068

23. Sanikidze V.M. and Sardzhevladze L.F. (1973). Terms of tea cuttings. Journal "Subtropical cultures", №3, (1973), pp:57-64. 